\title{
A coincidence and common fixed point theorem for subsequentially continuous hybrid pairs of maps satisfying an implicit relation
}

\author{
Said Beloul and Anita Tomar
}

\begin{abstract}
In this paper, we introduce the notion of subsequential continuity for a hybrid pair of maps and combine this concept with compatibility, to establish a coincidence and common fixed point theorem for a hybrid quadruple of maps. Our main result also demonstrates that several fixed point theorems can be unified using implicit relations. We also give two examples in support our results.
\end{abstract}

\section{INTRODUCTION}

Hybrid fixed point theory, which is the realm of common fixed point theorems for single valued and multivalued maps in metric spaces, has prospective applications in functional inclusions, optimization theory, fractal graphics and discrete dynamics for set valued operators. Since the work of Nadler [18], many authors gave generalizations of contraction principle of Banach for multivalued and hybrid pair of maps (for instance [1, 2, 4, 5, 14, 15, 25, 27, 28] and reference there in). Jungck [10] introduced the concept of compatibility for a pair of self maps, which has been extended to hybrid pair of maps by Kaneko and Sessa [15] and Beg and Azam [5] when $T: X \rightarrow C B(X)$. Recently, Bouhadjera and Godet Tobie [7] introduced subsequential continuity which is weaker than the reciprocal continuity introduced by pant [19]. In fact every non-vacuously pair of reciprocally continuous maps is naturally subsequentially continuous. However subsequentially continuous maps are neither sequentially continuous nor reciprocally continuous. For a brief development of variants of continuity and the relation between them one may refer to Tomar and Karapinar [30].

In this paper, we introduce the notion of subsequential continuity for a hybrid pair of maps and combine this concept with compatibility, in order to establish a common fixed point theorem for two hybrid pairs of maps satisfying implicit relation in metric spaces. Our main result also demonstrates

2010 Mathematics Subject Classification. Primary: 47H10, 54H25.

Key words and phrases. Common fixed point, subsequentially continuous, compatible mappings, implicit relation. 
that several fixed point theorems can be unified using implicit relations introduced by Popa [25].

\section{Preliminaries}

Let $(X, d)$ be a metric space, and let $C B(X)$ be a set of non empty, closed and bounded subsets of $X$. The Hausdorff metric is defined as:

$$
H(A, B)=\max \left\{\sup _{a \in A} d(a, B), \sup _{b \in B} d(b, A)\right\},
$$

for all $A, B \in C B(X)$ and $d(a, B)=\inf \{d(a, b), b \in B\}$.

If $A=\{a\}$ and $B=\{b\}$ (singleton), we write $H(A, B)=d(a, b)$.

Definition 2.1. Maps $f: X \rightarrow X$ and $S: X \rightarrow C B(X)$ on metric space $(X, d)$ are compatible if and only if for all $x \in X, f S x \in C B(X)$ and

$$
\lim _{n \rightarrow \infty} H\left(f S x_{n}, S f x_{n}\right)=0,
$$

whenever $\left\{x_{n}\right\}$ is a sequence in $X$ such that $\lim _{n \rightarrow \infty} S x_{n}=M \in C B(X)$ and $\lim _{n \rightarrow \infty} f x_{n}=t \in M$.

Example 2.1. Let $X=[0,2]$ endowed with the euclidian metric. Define $f$ and $S$ as follows:

$$
f x=\left\{\begin{array}{ll}
0, & 0 \leq x<1, \\
\frac{x+1}{2}, & 1 \leq x \leq 2,
\end{array} \quad S x= \begin{cases}\left\{\frac{1}{2}\right\}, & 0 \leq x<1, \\
{[1, x],} & 1 \leq x \leq 2 .\end{cases}\right.
$$

Consider a sequence $\left\{x_{n}\right\}$ defined by

$$
x_{n}=1+\frac{1}{n}, \text { for all } n \geq 1,
$$

we have

$$
\begin{gathered}
f x_{n}=1+\frac{1}{2 n} \rightarrow 1 \text { as } n \rightarrow \infty, \\
S x_{n}=\left[1,1+\frac{1}{n}\right] \rightarrow\{1\} \text { as } n \rightarrow \infty,
\end{gathered}
$$

and $\lim _{n \rightarrow \infty} H\left(f S x_{n}, S f x_{n}\right)=0$, so pair $(f, S)$ is compatible.

Singh and Mishra [29] introduced the notion of reciprocal continuity for a hybrid pair of single valued and multivalued maps as follows:

Definition 2.2. Maps $f: X \rightarrow X$ and $S: X \rightarrow C B(X)$ on metric space $(X, d)$ are reciprocally continuous on $X$ (resp. at $t \in X)$ if and only if $f S x \in B(X)$ for each $x \in X$ (resp. $f S t \in B(X))$ and

$$
\lim _{n \rightarrow \infty} f S x_{n}=f M, \lim _{n \rightarrow} S f x_{n}=S t,
$$

where $\left\{x_{n}\right\}$ is a sequence in $X$ such that $\lim _{n \rightarrow \infty} S x_{n}=M \in B(X), \lim _{n \rightarrow \infty} f x_{n}=$ $t \in M$. 
Definition $2.3([7])$. Maps $f$ and $g$ on a metric space $(X, d)$ are said to be subsequentially continuous if there exists a sequence $\left\{x_{n}\right\}$ such that:

$$
\lim _{n \rightarrow \infty} f x_{n}=\lim _{n \rightarrow \infty} g x_{n}=z,
$$

for some $z \in X$ and

$$
\begin{aligned}
& \lim _{n \rightarrow \infty} f g x_{n}=f z \\
& \lim _{n \rightarrow \infty} g f x_{n}=g z .
\end{aligned}
$$

Let $\mathcal{F}$ is the set of of all continuous functions $F: \mathbb{R}_{+}^{6} \rightarrow \mathbb{R}_{+}$satisfying the following conditions:

$\left(F_{1}\right): F$ non increasing in $t_{5}, t_{6}$.

$\left(F_{2}\right)$ : For any $v>0$, we have $F(v, u, 0,0, u, u)>0$, for every $u>0$.

Example 2.2.

$$
F\left(t_{1}, t_{2}, t_{3}, t_{4}, t_{5}, t_{6}\right)=t_{1}-\alpha \max \left\{t_{2}, t_{3}, t_{4}, t_{5}, t_{6}\right\},
$$

where $0 \leq \alpha<1$.

\section{Example 2.3.}

$F\left(t_{1}, t_{2}, t_{3}, t_{4}, t_{5}, t_{6}\right)=t_{1}-\alpha t_{2}-\beta \max \left\{t_{3}, t_{4}\right\}-\gamma \max \left\{t_{2}+t_{3}, t_{4}\right\}-\delta \max \left\{t_{5}, t_{6}\right\}$, where $\alpha, \beta, \gamma>0, \delta \geq 0$ and $\alpha+\beta+\gamma+\delta<1$.

\section{Example 2.4.}

$$
F\left(t_{1}, t_{2}, t_{3}, t_{4}, t_{5}, t_{6}\right)=t_{1}-\alpha \max \left\{t_{2}, t_{3}, t_{4}\right\}-(1-\alpha)\left\{a t_{5}+b t_{6}\right\},
$$

where $0 \leq \alpha<1, a, b \geq 0$ and $a+b<1$.

Example 2.5.

$$
F\left(t_{1}, t_{2}, t_{3}, t_{4}, t_{5}, t_{6}\right)=t_{1}-\phi\left(t_{2}, t_{3}, t_{4}, t_{5}, t_{6}\right),
$$

where $\phi: \mathbb{R}_{+}^{5} \rightarrow \mathbb{R}_{+}$is an upper semi continuous function such that $\phi(0)=0$ and $\phi(t, 0,0, t, t)<t$ for each $t>0$.

Example 2.6.

$$
F\left(t_{1}, t_{2}, t_{3}, t_{4}, t_{5}, t_{6}\right)=t_{1}-\psi\left(\max \left\{t_{2}, t_{3}, t_{4}, t_{5}, t_{6}\right\}\right),
$$

where $\psi: \mathbb{R}_{+} \rightarrow \mathbb{R}_{+}$is an increasing function such $\psi(t)<t$ for each $t>0$.

Example 2.7.

$$
F\left(t_{1}, t_{2}, t_{3}, t_{4}, t_{5}, t_{6}\right)=t_{1}-\left[a t_{2}^{p}+b t_{3}^{p}+c t_{4}^{p}\right]^{\frac{1}{p}}-d t_{5} t_{6}
$$

, where $a>(1+d)^{p}, \quad d \geq 0,0 \leq c, b<1, p \in \mathbb{N}$. 


\section{MAIN RESULTS}

First, we introduce subsequentially continuity for a hybrid pair of single valued and multivalued maps.

Definition 3.1. Maps $f: X \rightarrow X$ and $S: X \rightarrow C B(X)$ on metric space $(X, d)$ are subsequentially continuous if there exists a sequence $\left\{x_{n}\right\}$ such that

$$
\lim _{n \rightarrow \infty} S x_{n}=M \in C B(X) \text { and } \lim _{n \rightarrow \infty} f x_{n}=z \in M,
$$

for some $z \in X$ and $\lim _{n \rightarrow \infty} f S x_{n}=f M, \lim _{n \rightarrow \infty} S f x_{n}=S z$.

Notice that continuity or reciprocal continuity implies subsequential continuity, but the converse may be not true.

Example 3.1. Let $X=[0,2]$ and $d$ the Euclidian metric, we define $f, S$ by

$$
f x=\left\{\begin{array}{ll}
2-x, & 0 \leq x \leq 1, \\
\frac{1}{2}, & 1<x \leq 2,
\end{array} \quad S x= \begin{cases}{[0, x],} & 0 \leq x \leq 1, \\
{[x-1, x],} & 1<x \leq 2 .\end{cases}\right.
$$

We consider a sequence $\left\{x_{n}\right\}$ defined for each $n \geq 1$ by: $x_{n}=1-\frac{1}{n}$. Clearly $\lim _{n \rightarrow \infty} f x_{n}=1 \in[0,1]$ and $\lim _{n \rightarrow \infty} S x_{n}=[0,1] \in C B(X)$. Also we have:

$$
\lim _{n \rightarrow \infty} f S x_{n}=\lim _{n \rightarrow \infty}\left[1+\frac{1}{n}, 2\right]=[1,2]=f[0,1],
$$

and

$$
\lim _{n \rightarrow \infty} S f x_{n}=\lim _{n \rightarrow \infty}\left[\frac{1}{n}, 1+\frac{1}{n}\right]=[0,1]=S 1,
$$

then $(f, S)$ is subsequentially continuous.

On the other hand, consider a sequence $\left\{y_{n}\right\}$ defined for all $n \geq 1$ by: $y_{n}=1+\frac{1}{n}$, we have

$$
\lim _{n \rightarrow \infty} f y_{n}=\frac{1}{2} \in[0,1], \text { and } \lim _{n \rightarrow \infty} S y_{n}=[0,1] \in C B(X),
$$

however

$$
\lim _{n \rightarrow \infty} f S y_{n}=\lim _{n \rightarrow \infty} f\left(\left[\frac{1}{n}, 1+\frac{1}{n}\right]\right) \neq f([0,1]),
$$

then $f$ and $S$ are never reciprocally continuous. Also $\lim _{n \rightarrow \infty} H\left(f S x_{n}, S f x_{n}\right) \neq$ 0 , i.e, pair $(f, S)$ is not compatible.

Example 3.2. Let $X=\mathbb{R}_{+}$endowed with the euclidian metric, we define $f, S$ by

$$
f x=\left\{\begin{array}{ll}
\frac{x+2}{2}, & 0 \leq x \leq 2, \\
x-1, & x>2,
\end{array} \quad S x= \begin{cases}\{2\}, & 0 \leq x \leq 2, \\
\{1\}, & x>2\end{cases}\right.
$$


Let $\left\{x_{n}\right\}$ be sequence such that $x_{n}=2+e^{-n}$, for each $n \geq 1$. Clearly $\lim _{n \rightarrow \infty} f x_{n}=1 \in\{1\}$ and $\lim _{n \rightarrow \infty} S x_{n}=\{1\} \in C B(X)$. Also we have:

$$
\lim _{n \rightarrow \infty} f S x_{n}=f\{1\}
$$

and

$$
\lim _{n \rightarrow \infty} S f x_{n}=\{2\}=S 1,
$$

then $(f, S)$ is subsequentially continuous.

Now, let $\left\{y_{n}\right\}$ be a sequence such $y_{n}=\frac{1}{n}$, for each $n \geq 1$. We have

$$
\lim _{n \rightarrow \infty} f y_{n}=2 \in\{2\}, \text { and } \lim _{n \rightarrow \infty} S y_{n}=\{2\} \in C B(X),
$$

however

$$
\lim _{n \rightarrow \infty} S f y_{n}=\{1\} \neq S 2,
$$

then $f$ and $S$ are never reciprocally continuous.

Also $\lim _{n \rightarrow \infty} H\left(f S x_{n}, S f x_{n}\right)=0$, i.e, pair $(f, S)$ is compatible.

Remark 3.1. In Example 3.1 hybrid pair of maps is subsequentially continuous but is neither compatible nor reciprocal continuous. However in Example 3.2, hybrid pair is both subsequentially continuous and compatible but not reciprocally continuous. Hence subsequential continuity for a hybrid pair of single valued and multivalued maps is independent of compatibility. Further one may notice that continuity and reciprocal continuity for hybrid pair of maps implies subsequential continuity, but the converse may be not be true.

Theorem 3.1. Let $f, g: X \rightarrow X$, be single valued maps and $S, T: X \rightarrow$ $C B(X)$ multivalued maps on a metric space $(X, d)$ such that the pairs $(f, S),(g, T)$ are subsequentially continuous and compatible for all $x, y \in X$. Then the hybrid pair of maps $(f, S)$ and $(g, T)$ have a coincidence point. Moreover $f, g, S$ and $T$ have a common fixed point in $X$ provided that maps satisfy:

$$
\begin{aligned}
& F(H(S x, T y), d(f x, g y), d(f x, S x), \\
& d(g y, T y), d(f x, T y), d(g y, S x)) \leq 0,
\end{aligned}
$$

for all $x, y \in X$, where $F \in \mathcal{F}$.

Proof. Since $f$ and $S$ are subsequentially continuous, there exists a sequence $\left\{x_{n}\right\}$ in $X$ such that

$$
\lim _{n \rightarrow \infty} f S x_{n}=f M \text { and } \lim _{n \rightarrow \infty} S f x_{n}=S z
$$

and $\lim S x_{n}=M \in C B(X), \lim _{n \rightarrow \infty} f x_{n}=z \in M$.

Also, the compatibility of the pair $(f, S)$ implies that

$$
\lim _{n \rightarrow \infty} H\left(f S x_{n}, S f x_{n}\right)=H(f M, S z)=0,
$$


i,e, $f M=S z$, and $z$ is a coincidence point for $f$ and $S$.

Similarly, since pair $(g, T)$ is subsequentially continuous and compatible $g N=T t$. Hence $t$ is a coincidence point for $g$ and $T$.

We claim $f z=g t$, if not by using (1) we get:

$$
F(H(S z, T t), d(f z, g t), d(f z, S z), d(g y, T t), d(f z, T t), d(g t, S z)) \leq 0,
$$

or

$$
F(H(S z, T t), d(f z, g t), 0,0, d(f z, T t), d(g t, S z)) \leq 0 .
$$

Since $F$ is non increasing in $t_{5}, t_{6}$, we get

$$
F(H(g z, g t), d(f z, g t), 0,0, d(f z, g t), d(g t, f z)) \leq 0
$$

which is a contradiction with $\left(F_{2}\right)$, hence $f z=g$.

Nextly, we show that $z=f z$, if not by using (1) we get:

$F\left(H\left(S x_{n}, T t\right), d\left(f x_{n}, g t\right), d\left(f x_{n}, S x_{n}\right), d(g t, T t), d\left(f x_{n}, T t\right), d\left(g t, S x_{n}\right)\right) \leq 0$.

Letting $n \rightarrow \infty$, we get:

$$
F(H(M, T t), d(z, f z), 0,0, d(z, T t), d(f z, M)) \leq 0 .
$$

Since $z \in M$ and $f z \in S z$,

$$
F(d(z, f z), d(z, f z), 0,0, d(z, f z), d(z, f z)) \leq 0,
$$

which is a contradiction, hence $z=f z \in S z$ and $z$ is common fixed point of $f$ and $S$.

Now we prove $z=t$, if not by using (1) we get:

$$
\begin{aligned}
& F\left(H\left(S x_{n}, T y_{n}\right), d\left(f x_{n}, g y_{n}\right), d\left(f x_{n}, S x_{n}\right),\right. \\
& \left.d\left(g y_{n}, T y_{n}\right), d\left(f x_{n}, T y_{n}\right), d\left(g y_{n}, S x_{n}\right)\right) \leq 0 .
\end{aligned}
$$

Letting $n \rightarrow \infty$, we get:

$$
F(H(M, N), d(z, t), 0,0, d(z, N), d(t, M)) \leq 0 .
$$

Since $z \in M$ and $t \in N$, we get:

$$
F(H(z, t), d(z, t), 0,0, d(z, t), d(z, t)) \leq 0,
$$

which is a contradiction. Hence $z=t$, i.e., $z$ is common fixed point for $f, g, S$ and $T$.

If $f=g$ and $S=T$, we obtain the following corollary:

Corollary 3.1. Let $f: X \rightarrow X$ be a single valued map and $S: X \rightarrow C B(X)$ be multivalued map on a metric space $(X, d)$ such that the hybrid pair $(f, S)$ is subsequentially continuous and compatible for all $x, y \in X$. Then the hybrid pair of maps $(f, S)$ has a coincidence point. Moreover $f$ and $S$ have a common fixed point in $X$ provided that maps satisfy:

$$
F(H(S x, S y), d(f x, f y), d(f x, S x), d(f y, S y), f(f x, S y), d(f y, S x)) \leq 0,
$$
for $x, y \in X$, where $F \in \mathcal{F}$. 
Example 3.3. Let $X=\mathbb{R}_{+}$endowed with the euclidian metric, define maps $f$ and $S$ as follows:

$$
f x=\left\{\begin{array}{ll}
2, & 0 \leq x<1, \\
\frac{x+1}{2}, & x \geq 1,
\end{array} \quad S x= \begin{cases}{\left[0, \frac{3}{4}\right],} & 0 \leq x<1, \\
\{1\}, & x \geq 1 .\end{cases}\right.
$$

We consider a sequence $\left\{x_{n}\right\}$ defined for each $n \geq 1$ by:

$x_{n}=1+\frac{1}{n}$, clearly $\lim _{n \rightarrow \infty} S x_{n}=\{1\}$ and $\lim _{n \rightarrow \infty} f x_{n}=1 \in\{1\}$. So:

$$
\lim _{n \rightarrow \infty} f S x_{n}=\{1\}=f\{1\}, \quad \lim _{n \rightarrow \infty} S f x_{n}=S\left(1+\frac{1}{2 n}\right)=S 1,
$$

then $\{f, S\}$ is subsequentially continuous. Also, we have:

$$
\lim _{n \rightarrow \infty} H\left(f S x_{n}, S f x_{n}\right)=H(\{1\},\{1\})=0,
$$

so $f$ and $S$ are compatible.

Now, by taking

$$
F\left(t_{1}, t_{2}, t_{3},, t_{4}, t_{5}, t_{6}\right)=t_{1}-\frac{4}{5} \max \left\{t_{2}, t_{3}, t_{4}, t_{5}, t_{6}\right)
$$

in corollary 3.1, and we show that the following inequality hold.We have the following cases:

$$
H(S x, S y) \leq \frac{4}{5} \max \{d(f x, f y), d(f x, S x), d(f y, S y), d(f x, S y), d(f y, S x)\} .
$$

(1) For $x, y \in[0,1)$, we have $H(S x, S y)=0$, so obviously the inequality holds.

(2) For $x \in[0,1)$ and $y \geq 1$, we have

$$
H(S x, S y)=\frac{1}{4} \leq \frac{4}{5}=\frac{4}{5} d(f x, S x) .
$$

(3) For $x \in[1, \infty)$ and $y \in[0,1)$, we have

$$
H(S x, S y)=\frac{1}{4} \leq \frac{4}{5}=\frac{4}{5} d(f y, S y) .
$$

(4) For $x, y \in[1, \infty)$, we have $H(S x, S y)=0$, so the inequality holds.

Hence all hypotheses of corollary 3.1 are satisfied and the point 1 is a common fixed for $f$ and $S$.

Corollary 3.2. Let $f, g: X \rightarrow X$ be single valued maps and let $S, T: X \rightarrow$ $C B(X)$ be multivalued maps on a metric space $(X, d)$ such that the hybrid pairs $(f, S)$ and $(g, T)$ are subsequentially continuous and compatible for all $x, y \in X$. Then the hybrid pair of maps $(f, S)$ and $(g, T)$ has a coincidence point. Moreover $f, g, S$ and $T$ have a common fixed point in $X$ provided that maps satisfy

$$
H(S x, T y) \leq \alpha \max \{d(f x, g y), d(f x, S x), d(g y, T y), f(f x, T y), d(g y, S x)\},
$$

where $0 \leq \alpha<1$. 
Proof. Proof follows immediately on taking

$$
F\left(t_{1}, t_{2}, t_{3}, t_{4}, t_{5}, t_{6}\right)=t_{1}-\alpha \max \left\{t_{2}, t_{3}, t_{4}, t_{5}, t_{6}\right\}
$$

in theorem 3.1, where $0 \leq \alpha<1$.

Corollary 3.3. Let $f, g: X \rightarrow X$ be single valued maps and let $S, T: X \rightarrow$ $C B(X)$ be multivalued maps on a metric space $(X, d)$ such that the hybrid pairs $(f, S)$ and $(g, T)$ are subsequentially continuous and compatible for all $x, y \in X$. Then the hybrid pair of maps $(f, S)$ and $(g, T)$ has a coincidence point. Moreover $f, g, S$ and $T$ have a common fixed point in $X$ provided that maps satisfy

$$
H(S x, T y) \leq \phi(d(f x, g y), d(f x, S x), d(g y, T y), d(f x, T y), d(g y, S x)),
$$

where $\phi: \mathbb{R}_{+}^{5} \rightarrow \mathbb{R}_{+}$is an upper semicontinuous function such $\phi(0)=0$ and $\phi(t, 0,0, t, t)<t$ for each $t>0$.

Proof. Proof follows immediately on taking

$$
F\left(t_{1}, t_{2}, t_{3}, t_{4}, t_{5}, t_{6}\right)=t_{1}-\phi\left(t_{2}, t_{3}, t_{4}, t_{5}, t_{6}\right)
$$

in theorem 3.1, where $\phi: \mathbb{R}_{+}^{5} \rightarrow \mathbb{R}_{+}$is an upper semi continuous function such that $\phi(0)=0$ and $\phi(t, 0,0, t, t)<t$ for each $t>0$.

Corollary 3.4. Let $f, g: X \rightarrow X$ be single valued maps and let $S, T: X \rightarrow$ $C B(X)$ be multivalued maps on a metric space $(X, d)$ such that the hybrid pairs $(f, S)$ and $(g, T)$ are subsequentially continuous and compatible for all $x, y \in X$. Then the hybrid pair of maps $(f, S)$ and $(g, T)$ has a coincidence point. Moreover $f, g, S$ and $T$ have a common fixed point in $X$ provided that maps satisfy

$H(S x, T y) \leq \alpha \max \{d(f x, g y), d(f x, S x), d(g y, T y)\}-(1-\alpha)(a d(f x, T y)+$ $b d(g y, S x))$, where $0 \leq \alpha<1, a+b<1$.

Proof. Proof follows immediately on taking

$$
F\left(t_{1}, t_{2}, t_{3}, t_{4}, t_{5}, t_{6}\right)=t_{1}-\alpha \max \left\{t_{2}, t_{3}, t_{4}\right\}-(1-\alpha)\left\{a t_{5}+b t_{6}\right\}
$$

in theorem 3.1, where $0 \leq \alpha<1, a, b \geq 0$ and $a+b<1$.

Example 3.4. Let $X=[0,4]$ endowed with the euclidian metric, define maps $f, g, S$ and $T$ as follows:

$$
\begin{gathered}
f x=\left\{\begin{array}{ll}
\frac{x+2}{2}, & 0 \leq x \leq 2, \\
0, & 2<x \leq 4,
\end{array} \quad g x= \begin{cases}x+1, & 0 \leq x \leq 1, \\
2, & x=2, \\
4, & 2<x \leq 4,\end{cases} \right. \\
S x=\left\{\begin{array}{ll}
{[2,4-x],} & 0 \leq x \leq 2, \\
\left\{\frac{7}{4}\right\}, & 2<x \leq 4,
\end{array} \quad T x= \begin{cases}\{2\}, & 0 \leq x \leq 2, \\
{[0,1],} & 2<x \leq 4 .\end{cases} \right.
\end{gathered}
$$


We consider a sequence $\left\{x_{n}\right\}$ defined for each $n \geq 1$ by: $x_{n}=2-\frac{1}{n}$, clearly $\lim _{n \rightarrow \infty} S x_{n}=\{2\}$ and $\lim _{n \rightarrow \infty} f x_{n}=2 \in\{2\}$, also we have:

$$
\begin{gathered}
\lim _{n \rightarrow \infty} f S x_{n}=\{2\}=f\{2\} \\
\lim _{n \rightarrow \infty} S f x_{n}=S 2=\{2\} .
\end{gathered}
$$

Also $\lim _{n} H\left(f S x_{n}, S f x_{n}\right)=0$. Hence $(f, S)$ is subsequentially continuous and compatible.

For $g$ and $T$, consider a sequence $\left\{y_{n}\right\}$ defined by: $y_{n}=2-e^{-n}$, for all $n \geq 1$. It is clear that $\lim _{n \rightarrow \infty} g y_{n}=2, \lim _{n \rightarrow \infty} T x_{n}=\{2\}$, and $\lim _{n \rightarrow \infty} g T y_{n}=$ $g\{2\}=\{2\}, \lim _{n \rightarrow \infty} T g y_{n}=T 2=\{2\}$,

$$
\lim _{n \rightarrow \infty} H\left(g T y_{n} T g y_{n}\right)=0,
$$

i.e., $(g, T)$ is subsequentially continuous and compatible.

By taking $\alpha=\frac{2}{3}$ and $a=b=\frac{1}{4}$ in corollary 3.4, we show that the inequality is satisfied. We have the following cases:

(1) For $x, y \in[0,2]$, we have $H(S x, T y)=0$, obviously inequality is satisfied.

(2) For $x \in[0,2]$ and $y \in(2,4]$, we have

$$
H(S x, T y)=1 \leq 2=\frac{2}{3} d(g y, T y)
$$

(3) For $x \in(2,4]$ and $y \in[0,2]$, we have

$$
H(S x, T y)=\frac{1}{4} \leq \frac{7}{6}=\frac{2}{3} d(f x, S x)
$$

(4) For $x, y \in(2,4]$, we have

$$
H(S x, T y)=\frac{3}{4} \leq \frac{8}{3}=\frac{2}{3} d(f x, g y),
$$

then all hypotheses of corollary 3.4 are satisfied and the point 2 is a common fixed for $f, g, S$ and $T$.

Remark 3.2. $\quad(i)$ Clearly subsequentially continuous and compatible hybrid quadruple of maps on a non- complete metric space have a coincidence point. However it is believed that a pair of continuous and commuting (and hence compatible) self maps of a complete metric space may not have a coincidence point. [ for instance, $g x=1+x$ and $f x=x, x \in[0,1)]$.

(ii) It is clear from Example 3.4 that $(S, f)$ and $(g, T)$ have different coincidence point,i.e., $f 2 \in S 2, f 2=g 1 \in T 1$.

(iii) None of the map is continuous in the illustrating Examples 3.3 and 3.4,i.e., continuity requirements of all the involved maps is completely relaxed whereas most of the earlier theorems require the continuity of at least one involved map. 
(iv) It is interesting to note that Examples 3.3 and 3.4 cannot be covered by all those coincidence and common fixed point theorems which require containment of range space and continuity of involved maps along with completeness (or closedness) of underlying space/subspace. Subsequential continuity is indeed a weaker notion than the continuity of one of the map. However containment of the range space of the involved maps is indispensable in [4] and [27]. Our results generalize, extend and improve multitude of common fixed point results existing in the literature (for instance Altun and Türkoğlu [4], Popa [25], Djoudi [8] and Sedghi et al.[27])and guarantee the existence of coincidence and common fixed point for discontinuous hybrid quadruple of maps in non-complete metric space without containment of the range space of involved maps.

\section{REFERENCES}

[1] M. Abbas and B. Rhoades, Common fixed point theorems for hybrid pairs of occasionally weakly compatible mappings satisfying generalized contractive condition of integral type, Fixed Point Theory Appl. Art. ID 54101,(2007) 9.

[2] A. Ahmed and M. Imdad,Some Common Fixed Point Theorems for Mappings and Multi-valued Mappings, J. Math. Anal. Appl 218,(1998),546-560.

[3] M. A. Al-Thagafi and N. Shahzad, Generalized I-nonexpansive selfmaps and invariant approximations, Acta Math. Sinica 24, no5, (2008), 867-876.

[4] I. Altun and D. Türkoğlu, Some fixed point theorems for weakly compatible multivalued mappings satisfying an implicit relation, Filomat 22 (1)(2008), 13-23.

[5] I. Beg, A. Azam, Fixed points of assymptotically regular multivalued mappings, J. Austral. Math. Sco. 53 (1992), 313-326.

[6] S. Beloul, A Common Fixed Point Theorem for Weakly Compatible Multi-Valued Mappings Satisfying Strongly Tangential Property,math.moravica vol 18(2),(2014), 63-72.

[7] H. Bouhadjera and C. Godet. Thobie, Common fixed point theorems for pairs of subcompatible maps, arXiv:0906.3159v1 [math.FA].(2009).

[8] A. Djoudi, A unique common fixed point for compatible mappings of type (B) satisfying an implicit relation, Demonstratio Math. 36 (2003), 763-770.

[9] M. Imdad, J. Ali and M. Tanveer, Remarks on some recent metrical common fixed point theorems, Appl. Math. Lett. 24(2011), 1165ï£1169.

[10] G. Jungck, Commuting mappings and fixed points, Amer. Math. Monthly, 83(4)(1976), 261-263.

[11] G. Jungck,Compatible mappings and common fixed points, Internat. J. Math. and Math. Sci, 9(1986), 771-779.

[12] G. Jungck, P.P. Murthy and Y.J. Cho, Compatible mappings of type (A) and common fixed points, Math. Japon., 38 (1993) 381-390

[13] G. Jungck, Common fixed points for noncontinuous nonself maps on nonmetric spaces, Far East J. Math. Sci. 4, no 2 (1996), 199-215.

[14] G. Jungck, B.E. Rhoades,Fixed point theorems for occasionally weakly compatible mappings, Fixed Point Theory 9 (2008) 383-384.

[15] H. Kaneko, S. Sessa, Fixed point theorems for compatible multi-valued and singlevalued mappings, Internat. J. Math. Math. Sci. VOL. 12 NO 2(1989), 257-262. 
[16] W. Liu, J. Wu and Z. Li, Common fixed points of single-valued and multi-valued maps,Internat. J. Math. Math. Sci., 19(2005), 3045-3055.

[17] Liu, Li-Shan, Common fixed points of a pair of single-valued mappings and a pair of set-valued mappings (Chinese), Qufu Shifan Daxue Xuebao Ziran Kexue Ban, 18(1992), no. 1, 6-10.

[18] S. B. Nadler, Multivalued contraction mappings, Pacific J. Math. 30 (1969), 475-488.

[19] R. P. Pant, A common fixed point theorem under a new condition, Indian J. Pure Appl. Math, no. 2, 30 (1999), 147-152.

[20] H. K. Pathak and M. S. Khan, Compatible mappings of type (B) and common fixed point theorems of Gregus type, Czechoslovak Math. J., 45 (120) (1995), 685-698.

[21] H. K. Pathak, Y. J. Cho, S. M. Kang and B. S. Lee, Fixed point theorems for compatible mappings of type $(P)$ and application to dynamic programming, Le Matematiche (Fasc. I), 50 (1995), 15-33.

[22] H. K. Pathak, Y.J. Cho, S. M. Khan, B. Madharia, Compatible mappings of type (C) and common fixed point theorems of Gregus type, Demonstratio Math. 31 (3) (1998) 499-518.

[23] H. K. Pathak, N. Shahzad, Gregus type fixed point results for tangential mappings satisfying contractive conditions of integral type, Bull. Belg. Math. Soc. Simon Stevin 16 (2) (2009) 277-288.

[24] V. Popa,A common fixed point theorem of weakly commuting mappings,Inst. Math. (Beograd) (N. S) 47, 61 (1990), 132-136

[25] V.Popa, A general fixed point theorem for four weakly compatible mappings satisfying an implicit relation, Filomat n 19 (2005), 45-51.

[26] R. A. Rashwan and Magdy A. Ahmed, Common fixed points of Gregus type multivalued mappings, Archivum Mathematicum, Vol. 38 (2002), No. 1, 37-47.

[27] S. Sedghi, I. Altun, N. Shobe,A fixed point theorem for multi-maps satisfying an implicit relation on metric spaces,Appl. Anal. Discrete Math. 2 (2008), 189-196.

[28] S. Sessa, On a weak commutativity condition of mappings in fixed point considerations, Publ. Inst. Math. Beograd 32 (46) (1982), 149-153.

[29] S. L. Singh and S. N. Mishra, Coincidence and fixed points of reciprocally continuous and compatible hybrid maps, Int. J. Math. Math. Sci.10(2002), 627-635.

[30] A. Tomar and E.Karapinar, On variants of continuity and existence of fixed point via meir-keeler contractions in MC-spaces, J. Adv. Math. Stud.,Vol. 9(2), 2016, 348-359.

Said Beloul

Department of Mathematics

Exact Sciences Faculty

UNIVERSITY OF EL-OUED

P.O. Box 789 El-Oued39000

Algeria

E-mail address: beloulsaid@gmail.com

\author{
Anita Tomar \\ Government P.G. College Dakpathar \\ Dehradun (UtTarakHand) \\ INDIA \\ E-mail address: anitatmr@yahoo.com
}

